Bundesgesundheitsbl - Gesundheitsforsch Gesundheitsschutz 2004 • 47:1033-1042 DOI 10.1007/s00103-004-0934-0

๑) Springer Medizin Verlag 2004

T. Lampert · M. Thamm • Robert Koch-Institut, Berlin

\title{
Soziale Ungleichheit des Rauchverhaltens in Deutschland
}

wirksame und nachhaltige Maßnahmen zu implementieren, muss an den generierenden und stabilisierenden Mechanismen der im Gesundheitszustand und Lebenserwartung zum Ausdruck kommenden sozialen Ungleichheit angesetzt werden. Einen wichtigen Anknüpfungspunkt gibt das Gesundheitsverhalten vor, das in sozialepidemiologischen Erklärungsmodellen als Verbindungsglied zwischen sozialstrukturellen Gegebenheiten und individuellen Gesundheitschancen berücksichtigt wird $[5,6]$. Wenngleich eine umfassende Betrachtung der gesundheitlichen Lebensweise unter Einbeziehung zugrunde liegender Einstellungen, Orientierungen und Werthaltungen angezeigt ist, lohnt die Fokussierung auf einzelne Verhaltensmuster, um spezifische Gesundheitsprobleme zu ermitteln und Handlungsbedarf abzuleiten. Ein hoher Stellenwert ist dabei dem Tabakkonsum und insbesondere dem Zigarettenrauchen beizumessen, zumal sich Zusammenhänge zu anderen Aspekten des Gesundheitsverhaltens, wie z. B. Ernährung, körperliche Aktivität oder Alkoholkonsum, herstellen lassen. Nach aktuellem Kenntnisstand kann das Rauchen als bedeutendstes einzelnes Gesundheitsrisiko und führende Ursache der vorzeitigen Sterblichkeit angesehen werden. $\mathrm{Zu}$ den Erkrankungen, die vermehrt bei Rauchern auftreten, zählen Herzinfarkt, Schlaganfall, Arteriosklerose, Bluthochdruckerkrankung, Lungenentzündung, chronische Bronchitis sowie bösartige Neubildungen der Lunge, der Mundhöhle, des Kehlkopfs und der Verdauungs- organe [7, 8]. In Deutschland sterben jedes Jahr zwischen 100.000 und 140.000 Menschen infolge von Krankheiten, die sich mit dem Rauchen in Verbindung bringen lassen; weltweit sind es wahrscheinlich mehr als 5 Millionen Menschen [9, 10].

\section{( In Deutschland sterben jährlich mehr als 100.000 Menschen an den Folgen des Rauchens}

Da die soziale Ungleichheit bei tabakassoziierten Erkrankungen und Gesundheitsstörungen einen deutlichen Niederschlag findet, liegt es nahe, das Rauchen in den Mittelpunkt sozialkompensatorischer gesundheitspolitischer Bemühungen zu rücken. Eine wesentliche Voraussetzung hierfür sind epidemiologische Analysen, die verdeutlichen, in welchen Bevölkerungsgruppen besonders viel geraucht wird und die außerdem Aufschluss über die sozialen Bestimmungsgründe der Initialisierung, Stabilisierung und Beendigung des Tabakkonsums geben. In Deutschland mangelt es bislang an aussagekräftigen Studien, obwohl durch den Mikrozensus des Statistischen Bundesamtes, die Repräsentativerhebung zum Gebrauch psychoaktiver Substanzen des Instituts für Therapieforschung, die Drogenaffinitätsstudie der Bundeszentrale für gesundheitliche Aufklärung und die bundesweit durchgeführten Gesundheitssurveys des Robert Koch-Instituts schon seit längerem bevölkerungsrepräsentative Daten zum Rauchen bereitgestellt werden. Die vorhandenen Forschungsergebnisse fügen sich jenungleichheit eine zentrale gesundheitspolitische Herausforderung dar $[3,4]$. Um 
Tabelle 1

Zentrale Untersuchungsvariablen der Analysen zur sozialen Ungleichheit des Rauchverhaltens $(\mathrm{n}=7.124)$

\begin{tabular}{|c|c|c|c|c|}
\hline Variable & Kategorien & $\begin{array}{l}\text { Fallzahl } \\
\text { (n) }\end{array}$ & $\begin{array}{l}\text { Stichprobe } \\
\text { [\%] }\end{array}$ & $\begin{array}{l}\text { Grundgesamt- } \\
\text { heit [\%] }\end{array}$ \\
\hline \multirow[t]{5}{*}{ Raucherstatus } & Tägliche Raucher & 1.853 & 26,0 & 26,3 \\
\hline & Gelegenheitsraucher & 442 & 6,2 & 6,2 \\
\hline & Exraucher & 1.483 & 20,8 & 21,7 \\
\hline & Nieraucher & 3.185 & 44,7 & 45,9 \\
\hline & Fehlende Werte & 161 & 2,3 & - \\
\hline \multirow[t]{4}{*}{ Sozialschicht } & Unterschicht & 1.621 & 22,8 & 23,0 \\
\hline & Mittelschicht & 3.817 & 53,6 & 55,4 \\
\hline & Oberschicht & 1.458 & 20,5 & 21,6 \\
\hline & Fehlende Werte & 228 & 3,2 & - \\
\hline \multirow[t]{4}{*}{ Schulbildung } & Volks- oder Hauptschule & 2.942 & 41,3 & 46,7 \\
\hline & Mittlere Reife & 2.272 & 31,9 & 30,1 \\
\hline & Abitur & 1.532 & 21,5 & 23,2 \\
\hline & Fehlende Werte & 378 & 5,3 & - \\
\hline \multirow[t]{4}{*}{ Äquivalenzeinkommen } & $<60 \%$ & 1.314 & 18,4 & 21,7 \\
\hline & $60-150 \%$ & 3.629 & 50,9 & 65,7 \\
\hline & $>150 \%$ & 617 & 8,7 & 12,6 \\
\hline & Fehlende Werte & 1.564 & 22,0 & - \\
\hline \multirow[t]{5}{*}{ Berufliche Stellung } & Niedrig & 1.042 & 14,6 & 16,7 \\
\hline & Einfach & 1.818 & 25,5 & 28,6 \\
\hline & Mittel & 1.969 & 27,6 & 32,2 \\
\hline & Höher & 1.370 & 19,2 & 22,4 \\
\hline & Fehlende Werte & 925 & 13,0 & - \\
\hline
\end{tabular}

a Gewichtet auf die 18-bis 79-jährige Wohnbevölkerung zum Zeitpunkt der Stichprobenziehung (ohne fehlende Werte). doch zu einem einheitlichen Bild zusammen und belegen ein ausgeprägtes soziales Gefälle mit den höchsten Raucheranteilen in Gruppen mit niedrigem Bildungsniveau und Berufsstatus $[11,12]$, einfacher manueller und einfacher Dienstleistungstätigkeit [13], geringem Einkommen und materieller Deprivation [14] sowie bei Arbeitslosen und Sozialhilfeempfängern [15]. Auch in den Rauchgewohnheiten schwangerer Frauen und von Müttern mit Kleinkindern sowie in der Passivrauchexposition zeichnen sich deutliche soziale Unterschiede ab $[16,17]$. In einer aktuellen Studie finden sich zudem Hinweise darauf, dass die soziale Polarisierung des Rauchverhaltens seit Mitte der 8oer-Jahre eher noch zu- als abgenommen hat [18].

Im Folgenden werden Daten des Bundes-Gesundheitssurveys 1998 für eine Analyse der sozialen Ungleichheit des Rauchverhaltens in Deutschland herangezogen, die nicht nur auf die Ermittlung gruppenspezifischer Raucheranteile zielt, sondern auch nach Unterschieden in der Aufhörwahrscheinlichkeit fragt. Zunächst wird die Schichtspezifität des Tabakkonsums untersucht und zu diesem Zweck auf einen mehrdimensionalen Index zurückgegriffen, der eine allgemeine Beschreibung des gesellschaftlichen Ungleichheitsgefüges gestattet. Im Anschluss werden Unterschiede nach Schulbildung, Einkommenssituation und beruflicher Stellung betrachtet, da eine an Einzelindikatoren orientierte Auswertung eher Rückschlüsse auf konkrete Problemlagen und Interventionspotenziale zulässt. Indem alters- und geschlechtsspezifische Variationen der sozialen Ungleichheit des Rauchverhaltens aufgezeigt werden, wird die Charakterisierung von Zielgruppen für die Tabakprävention und Tabakkontrollpolitik unterstützt.

\section{Datenbasis und Methoden}

Mit dem Bundes-Gesundheitssurvey 1998 steht eine umfassend belastbare Datenba- sis für epidemiologische Forschungen, $\mathrm{Ge}$ sundheitsberichterstattung und Gesundheitspolitik zur Verfügung. Für den vom Robert Koch-Institut konzipierten und zwischen Oktober 1997 und März 1999 durchgeführten Survey wurde eine mehrfach geschichtete 2-stufige Zufallsstichprobe gezogen, die zum Zeitpunkt der Stichprobengenerierung für die 18- bis 79-jährige Wohnbevölkerung Deutschlands repräsentativ war [19]. Ausgehend von der um die qualitätsneutralen Ausfälle bereinigten Bruttostichprobe entsprach die Teilnehmerzahl von 7.124 Personen einer Responserate von $62 \%$. Wie detaillierte NonResponderanalysen belegen, wird die Repräsentativität der Erhebungen durch den Stichprobenausfall nicht entscheidend beeinträchtigt [20].

Das Erhebungsprogramm des BundesGesundheitssurveys 1998 war thematisch breit angelegt und umfasste einen Befragungs- und einen Untersuchungsteil. Die Befragung basierte auf einem Selbstausfüllfragebogen und enthielt unter anderem Fragen zu Krankheiten, Beschwerden, gesundheitsbezogener Lebensqualität, Gesundheitsverhalten und Gesundheitsversorgung. Der Untersuchungsteil erstreckte sich auf ein ärztliches Interview zur Krankengeschichte und eine medizinische Untersuchung, die unter anderem die Messung von Blutdruck und Pulsfrequenz sowie die Feststellung von Körpergröße und Körpergewicht beinhaltete. Außerdem wurden Laboranalysen von Blutund Urinproben durchgeführt und an Substichproben Zusatzmodule zu ausgewählten Themen wie Ernährung, Folsäureversorgung, Umweltbelastungen und psychische Störungen realisiert [21].

Die Erhebung des Rauchverhaltens war Bestandteil der schriftlichen Befragung und erstreckte sich auf 5 Fragen zum aktiven Rauchen und 2 Fragen zur Passivrauchexposition. Für die Analyse der sozialen Ungleichheit des Rauchverhaltens wird, ausgehend von der Frage „Haben Sie früher geraucht oder rauchen Sie zurzeit?“, zwischen „täglichen Rauchern“, „Gelegenheitsrauchern“, „Exrauchern“ und „Nierauchern" unterschieden, wobei neben Zigarettenrauchern auch Konsumenten anderer Tabakwaren, wie z. B. Zigarren, Zigarillos oder Pfeifen, einbezogen werden. Wenn von „aktuellen Rauchern“ die Rede 
ist, sind sowohl die täglichen als auch die gelegentlichen Raucher gemeint. Betrachtet wird zudem die „Aussteigerquote“, die durch den Anteil der Exraucher an allen Personen, die jemals geraucht haben, bestimmt ist. Die Fragen zum Rauchen wurden von fast allen Studienteilnehmern beantwortet, sodass eine Verzerrung der Ergebnisse durch Item-Non-Response ausgeschlossen werden kann (• Tabelle 1).

Die Messung sozialer Ungleichheit orientiert sich am schichtungssoziologischen Zugang, der die Arbeitswelt als zentralen Entstehungsort und den Beruf als wichtigste Vermittlungsinstanz sozialer Vor- und Nachteilsbedingungen beschreibt. Für den Bundes-Gesundheitssurvey 1998 wurde ein Index entwickelt, der eine mehrdimensionale Betrachtung der Schichtzugehörigkeit erlaubt und damit die Möglichkeit der Kumulation von Ungleichheitsbedingungen berücksichtigt. Berechnet wird der Index als ungewichteter aggregierter Punktsummenscore auf der Basis von Angaben zum höchsten Schul- und Ausbildungsabschluss, Haushaltsnettoeinkommen sowie beruflichen Status. Durch Gruppierung der Punktwerte lässt sich zwischen einer Unter-, Mittel- und Oberschicht unterscheiden [22], wobei allerdings zu berücksichtigen ist, dass die Schichtgrenzen unter pragmatischen Gesichtspunkten gezogen werden und keine reale Entsprechung haben.

Als Einzelindikatoren der Schichtzugehörigkeit werden die Schulbildung, das äquivalenzbilanzierte Haushaltsnettoeinkommen und der berufliche Status betrachtet. Zur Erfassung der Schulbildung wird, ausgehend vom höchsten allgemein bildenden Schulabschluss, zwischen "Volksschul- oder Hauptschulabschluss", „Mittlerer Reife bzw. Abschluss der Polytechnischen Oberschule“ sowie „Abitur, Fachhochschulreife bzw. Abschluss der Erweiterten Oberschule“ differenziert. Für die Äquivalenzbilanzierung des Haushaltsnettoeinkommens, die neben der Anzahl auch das Alter der Haushaltsmitglieder als Approximation unterschiedlicher Einkommensbedarfe berücksichtigt, wurden Bedarfsgewichte entsprechend der alten OECD-Skala angesetzt (Haushaltsvorstand=1, 0 ; Haushaltsmitglieder $a b$ 15 Jahre $=0,7$; Haushaltsmitglieder unter 15 Jahre=0,5). Da das Haushaltsnettoein-

Bundesgesundheitsbl - Gesundheitsforsch - Gesundheitsschutz 2004 - 47:1033-1042

DOI 10.1007/s00103-004-0934-0

(c) Springer Medizin Verlag 2004

T. Lampert $\cdot$ M. Thamm

\section{Soziale Ungleichheit des Rauchverhaltens in Deutschland}

\section{Zusammenfassung}

Anhand von Daten des Bundes-Gesundheitssurveys 1998 wird der Einfluss alternativer Indikatoren sozialer Ungleichheit auf das Rauchverhalten der 18- bis 79-jährigen Bevölkerung in Deutschland untersucht. Danach rauchen die Angehörigen sozial benachteiligter Bevölkerungsgruppen nicht nur häufiger, innen fällt es offenbar auch schwerer, das Rauchen wieder aufzugeben. Besonders deutlich kommt dies im mittleren Lebensalter zum Ausdruck, während im höheren Alter nur noch schwache Unterschiede beobachtet werden können. Multivariate Analysen weisen bei Männern wie Frauen vor allem die Schulbildung als Determinante des Rauchverhaltens aus. Der
Einfluss des Einkommens und der beruflichen Stellung ist vergleichsweise gering. Die Ergebnisse sprechen dafür, dass die im Gesundheitsstatus und in der Lebenserwartung zutage tretende Chancenungleichheit zu einem Teil auf unterschiedliche Rauchgewohnheiten zurückzuführen ist, und unterstreichen damit die Notwendigkeit zielgruppenspezifischer Interventionen der Tabakprävention und Tabakkontrollpolitik.

\section{Schlüsselwörter}

Soziale Ungleichheit · Rauchen · Raucherentwöhnung · Tabakkontrollpolitik ·

Bundes-Gesundheitssurvey 1998

\section{Social inequality and smoking in Germany}

\begin{abstract}
Data of the National Health Survey 1998

are used to analyze the impact of alternative indicators of social inequality on smoking of the 18- to 79-year-old population in Germany. The members of socially disadvantaged groups not only smoke more frequently, they show also lower rates in smoking cessation. This is most obvious in middle age while only small differences can be observed at older ages. Multivariate analyses evidence primarily education as a determinant of smoking among men and women.
\end{abstract}

The effects of income and occupational status are fairly small. The results point out that health inequalities can partly be explained by different smoking habits and, therefore, underline the necessity of a differentiated and group-specific tobacco prevention and tobacco control policy.

\section{Keywords}

Social inequality $\cdot$ Smoking $\cdot$ Smoking cessation · Tobacco control policy · National Health Survey 1998 


\section{Originalien und Übersichtsarbeiten}
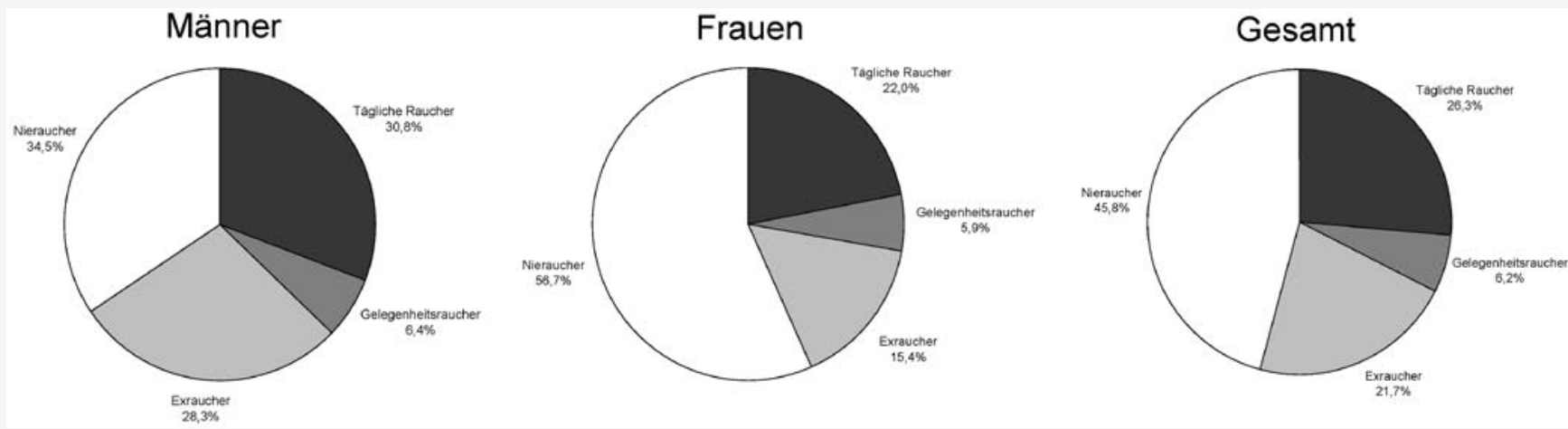

Abb. $1 \Delta$ Anteil der täglichen Raucher, Gelegenheitsraucher, Exraucher und Nieraucher bei Männern und Frauen $(n=6.963)$

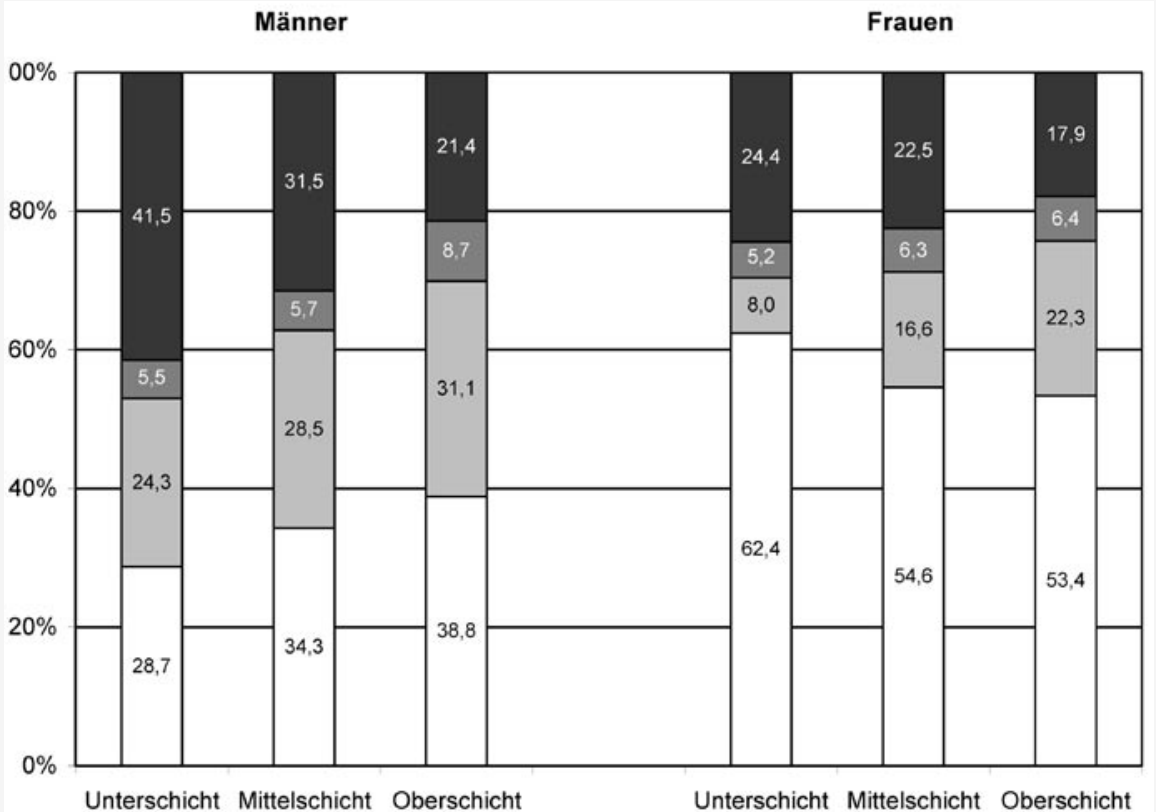

Sozialschicht

口Nieraucher $\quad$ Exraucher $\square$ Gelegenheitsraucher $\quad$ Tägliche Raucher

Abb. $2<$ Anteil der täglichen Raucher, Gelegenheitsraucher, Exraucher und Nieraucher nach Schichtzugehörigkeit (in \%, $n=6.963$ )

kommen im Bundes-Gesundheitssurvey 1998 kategorial erhoben wurde, gingen in die Berechnung statt genauer Einkommensbeträge die Mittelwerte der insgesamt 17 Einkommenskategorien, die anhand von Daten des Sozio-oekonomischen Panels (SOEP) desselben Jahres ermittelt wurden, ein. Für die Analysen werden 3 Einkommensgruppen unterschieden: „bis zu 60\%“, „60-150\%“ und „über $150 \%$ “ in Bezug auf das mittlere Äquivalenzeinkommen in der Bevölkerung, das 1998 bei 2.006 DM lag [23]. Die 60\%- und 150\%-Schwelle dienen dabei zur Abgrenzung von Niedrigeinkommen bzw. relativer Wohlhabenheit. Die Erhebung des beruflichen Status erfolgte mittels eines Standardinstruments, das für die Mikrozensus-Zusatzerhebung 1971 entwickelt wurde und seitdem in der Sozial- und Gesundheitsforschung breite Anwendung findet. Die insgesamt 19 Kategorien wurden zu 4 Statusgruppen zusammengefasst, wobei die Autonomie des beruflichen Handelns das ausschlaggebende $\mathrm{Zu}$ ordnungskriterium war [24]. Un- und angelernte Arbeiter haben demnach einen „niedrigen Berufsstatus“, während gelernte und Facharbeiter ebenso wie Angestellte mit einfacher Tätigkeit, Beamte im einfachen Dienst und selbstständige Landwirte durch einen „einfachen Berufssta- tus" ausgewiesen sind. Von einem „mittleren Berufsstatus“ wird im Zusammenhang mit Vorarbeitern und Kolonnenführern, Angestellten mit qualifizierter Tätigkeit, Beamten im mittleren Dienst und mithelfenden Familienangehörigen gesprochen. Die Gruppe mit „höherem Berufsstatus" wird von Industrie- und Werkmeistern, Angestellten mit hoch qualifizierter Tätigkeit oder umfassenden Führungsaufgaben, Beamten im gehobenen oder höheren Dienst sowie Selbstständigen und Angehörigen akademisch freier Berufe gebildet. Von einer häufig getroffenen Unterscheidung zwischen gehobenem und hohem Berufsstatus wurde an- 
gesichts der Stichprobengröße und der relativ geringen Besetzung der höchsten Statusgruppe abgesehen.

Die statistischen Analysen wurden mit dem Statistikprogramm SPSS 12.0 für Windows durchgeführt. Um repräsentative Aussagen treffen zu können, wurden die Ergebnisse anhand eines eigens für den Survey entwickelten Gewichtungsfaktors an die Bevölkerungsstruktur Deutschlands im Jahr 1998 angepasst.

\section{Ergebnisse}

In Deutschland rauchte im Jahr 1998 etwa ein Drittel der 18- bis 79-jährigen Bevölkerung. Rund $26 \%$ griffen täglich zur Zigarette oder einem anderen Tabakprodukt, während sich $6 \%$ als Gelegenheitsraucher bezeichneten. Weitere $22 \%$ gaben an, früher geraucht zu haben, sodass sich der Anteil der Personen, die jemals geraucht haben, auf insgesamt $54 \%$ beläuft. Frauen rauchten mit $28 \%$ seltener als Männer mit 37\%, wobei der Geschlechtsunterschied vor allem beim täglichen Tabakkonsum zu beobachten ist (• Abb. 1).

\section{( Im Jahr 1998 rauchte in}

Deutschland etwa ein Drittel der 18- bis 79-Jährigen.

Am häufigsten rauchten junge Erwachsene mit Prävalenzen von fast $50 \%$ bei Männern und über $40 \%$ bei Frauen (• Tabelle 2). Ab dem Alter 40 nimmt der Anteil der Raucher allmählich ab, bevor er sich ab dem Alter 60 sprunghaft verringert, und zwar bis auf $18 \%$ bei den 70 -jährigen und älteren Männern und $11 \%$ bei den gleichaltrigen Frauen. Am steigenden Anteil der Exraucher lässt sich ablesen, dass viele Männer im fortgeschrittenen Alter das Rauchen aufgeben, wofür neben Veränderungen der Lebensumstände, z. B. dem Ausscheiden aus dem Erwerbsleben und der Verlagerung der Freizeitgestaltung, auch alterskorrelierte gesundheitliche Beeinträchtigungen und daraus resultierende Anpassungen der Lebensweise verantwortlich sein dürften. Bei Frauen sind die im höheren Alter geringer ausfallenden Raucheranteile in erster Linie Ausdruck eines Kohorteneffekts. Viele der älteren Frauen haben nie geraucht, was auch im Zusammenhang mit dem in der ersten

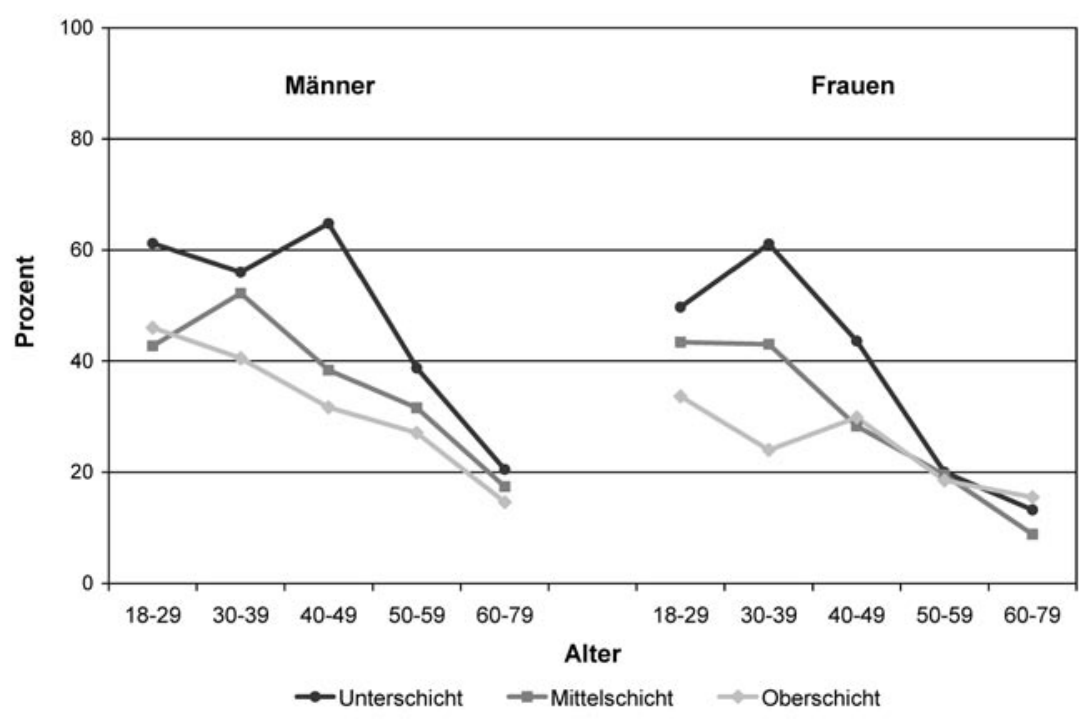

Abb. $3 \triangle$ Anteil der aktuellen Raucher nach Schichtzugehörigkeit und Alter (in \%, $\mathbf{n}=6.963$ )

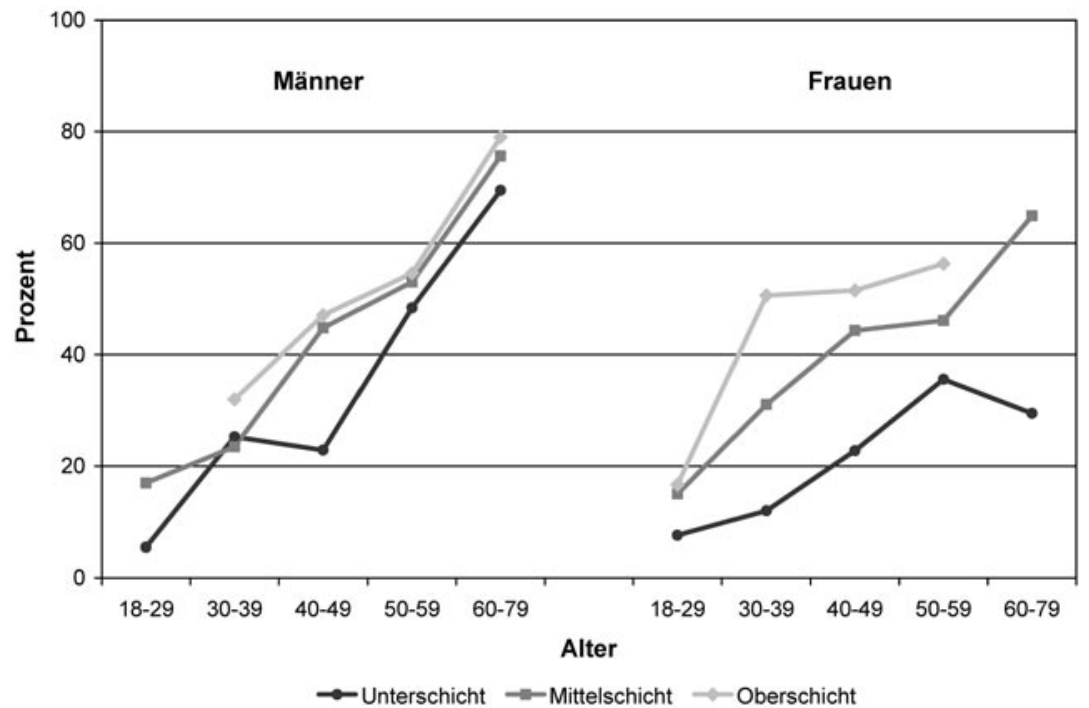

Abb. $4 \triangle$ Aussteigerquote nach Schichtzugehörigkeit und Alter (in \%, $\mathbf{n = 3 . 7 7 8 )}$

Hälfte des 20. Jahrhunderts vorherrschenden gesellschaftlichen Frauenbild zu sehen ist.

\section{Schichtspezifität \\ des Rauchverhaltens}

Darüber hinaus belegen die Daten des Bundes-Gesundheitssurveys 1998, dass es sich beim Rauchen um ein im hohen Maß schichtspezifisch geprägtes Verhaltensmuster handelt (• Abb. 2). Legt man den für den Survey entwickelten Schichtindex zugrunde, dann rauchen $47 \%$ der Männer aus der Unterschicht, aber nur $37 \%$ aus der Mittelschicht und 30\% aus der Oberschicht. Unter Einbeziehung der Anteile der Exraucher lässt sich feststellen, dass Männer, die der Unterschicht angehören, nicht nur häufiger rauchen, sondern auch seltener wieder aufhören. Bei Frauen sind ebenfalls schichtspezifische Unterschiede festzustellen, die allerdings schwächer ausgeprägt sind als bei Männern und zudem einem anderen Verteilungsmuster folgen, das durch einen geringeren Raucheranteil in der Oberschicht im Vergleich zu den anderen beiden Schichten, zwischen denen keine Unterschiede bestehen, charakterisiert ist. Die unterschiedlichen Anteile der 


\section{Originalien und Übersichtsarbeiten}

Tabelle 2

Anteil der täglichen Raucher, Gelegenheitsraucher, Exraucher und Nieraucher nach Alter und Geschlecht

(in \%, $\mathrm{n}=6.963$ )

\begin{tabular}{|c|c|c|c|c|c|c|c|c|c|c|c|c|}
\hline & \multicolumn{6}{|c|}{ Männer } & \multicolumn{6}{|l|}{ Frauen } \\
\hline & $18-29$ & $30-39$ & $40-49$ & $50-59$ & $60-79$ & Gesamt & $18-29$ & $30-39$ & $40-49$ & $50-59$ & $60-79$ & Gesamt \\
\hline Tägliche Raucher & 39,5 & 41,5 & 34,3 & 24,9 & 13,7 & 30,8 & 31,3 & 33,0 & 25,8 & 17,1 & 8,3 & 22,0 \\
\hline Gelegenheitsraucher & 8,9 & 7,6 & 5,8 & 6,1 & 3,9 & 6,4 & 12,2 & 8,0 & 5,1 & 2,7 & 2,8 & 5,9 \\
\hline Exraucher & 6,4 & 18,1 & 28,4 & 34,6 & 52,6 & 28,3 & 6,3 & 20,0 & 23,6 & 17,4 & 11,2 & 15,4 \\
\hline Nieraucher & 45,2 & 32,8 & 31,5 & 34,4 & 29,8 & 34,5 & 50,2 & 39,0 & 45,5 & 62,9 & 77,7 & 56,7 \\
\hline
\end{tabular}

Tabelle 3

Anteil der aktuellen Raucher nach Schulbildung, Äquivalenzeinkommen und beruflicher Stellung (in \%, $\mathrm{n}=6.963$ )

\begin{tabular}{|c|c|c|c|c|c|c|c|c|c|c|c|c|}
\hline & \multicolumn{6}{|l|}{ Männer } & \multicolumn{6}{|l|}{ Frauen } \\
\hline & $18-29$ & $30-39$ & $40-49$ & $50-59$ & $60-79$ & Gesamt & $18-29$ & $30-39$ & $40-49$ & $50-59$ & $60-79$ & Gesamt \\
\hline \multicolumn{13}{|l|}{ Schulbildung } \\
\hline $\begin{array}{l}\text { Volks- oder Haupt- } \\
\text { schule }\end{array}$ & 60,3 & 55,5 & 45,5 & 32,7 & 19,3 & 37,4 & 52,2 & 54,0 & 36,7 & 22,0 & 11,5 & 25,6 \\
\hline Mittlere Reife & 51,4 & 51,0 & 38,6 & 27,7 & 15,2 & 41,3 & 50,2 & 38,4 & 29,4 & 17,1 & 10,5 & 32,5 \\
\hline Abitur & 35,6 & 42,1 & 30,7 & 28,8 & 14,7 & 32,6 & 33,0 & 28,8 & 24,6 & 15,1 & 12,5 & 26,3 \\
\hline$\chi^{2}$-Test, Signifikanz & $p<0,001$ & $p<0,05$ & $p<0,01$ & n.s. & n.s. & $p<0,01$ & $p<0,001$ & $p<0,001$ & $p<0,05$ & n.s. & n.s. & $p<0,001$ \\
\hline \multicolumn{13}{|l|}{ Äquivalenzeinkommen } \\
\hline$<60 \%$ & 53,1 & 51,2 & 47,5 & 43,2 & 27,1 & 46,3 & 48,5 & 50,8 & 30,0 & 26,7 & 4,4 & 37,3 \\
\hline $60-150 \%$ & 44,6 & 48,4 & 38,8 & 28,1 & 16,2 & 35,1 & 40,8 & 38,9 & 31,1 & 17,0 & 12,5 & 26,5 \\
\hline$>150 \%$ & 47,3 & 45,7 & 24,6 & 29,4 & 16,0 & 32,4 & 39,0 & 32,2 & 42,1 & 25,0 & 6,8 & 29,1 \\
\hline$\chi^{2}$-Test, Signifikanz & n.s. & n.s. & $p<0,05$ & $p<0,05$ & n.s. & $p<0,001$ & n.s. & $p<0,01$ & n.s. & n.s. & n.s. & $p<0,001$ \\
\hline \multicolumn{13}{|l|}{ Berufliche Stellung } \\
\hline Niedrig & 54,5 & 66,0 & 53,8 & 40,8 & 23,5 & 47,1 & 55,6 & 59,1 & 40,6 & 26,7 & 9,6 & 30,4 \\
\hline Einfach & 49,4 & 49,3 & 50,3 & 39,4 & 16,5 & 40,8 & 47,4 & 42,9 & 30,9 & 16,9 & 12,9 & 26,8 \\
\hline Mittel & 49,5 & 48,6 & 26,5 & 22,2 & 16,2 & 31,7 & 40,2 & 36,3 & 26,0 & 17,8 & 8,1 & 25,8 \\
\hline Höher & 49,1 & 44,4 & 38,1 & 28,1 & 17,5 & 32,6 & 43,3 & 29,5 & 36,1 & 21,0 & 22,0 & 29,0 \\
\hline$\chi^{2}$-Test, Signifikanz & n.s. & $p<0,01$ & $p<0,001$ & $p<0,01$ & n.s. & $p<0,001$ & n.s. & $p<0,001$ & $p<0,05$ & n.s. & $p<0,05$ & n.s. \\
\hline
\end{tabular}

Exraucherinnen deuten an, dass auch bei Frauen die Angehörigen der höheren Sozialschichten häufiger das Rauchen wieder aufgeben.

\section{Beim Rauchen handelt es sich um ein im hohen Maß schichtspezifisch geprägtes Verhaltensmuster}

Eine nach Altersgruppen differenzierte Betrachtung verdeutlicht, dass sich der Schichtgradient im Rauchverhalten bei Männern wie Frauen im mittleren Lebensalter am stärksten abzeichnet (- Abb. 3). Mit zunehmendem Alter treten die schichtspezifischen Unterschiede im Rauchen schwächer hervor, und spätestens nach dem Übergang in den Ruhestand sind sie nicht mehr sichtbar. Die- ser Befund steht im Einklang mit den Ergebnissen britischer und skandinavischer Studien, die für den mittleren Lebensabschnitt stark ausgebildete soziale Unterschiede im Rauchverhalten und anderen verhaltenskorrelierten Gesundheitsrisiken berichten, die sich im höheren Alter sukzessive verringern $[25,26]$.

Ähnlich auffällige Schichtunterschiede lassen sich in Bezug auf die Aussteigerquote beobachten. In $\bullet$ Abb. 4 fehlen aufgrund geringer Fallzahlen die Angaben für 18- bis 29-jährige Männer und 60-jährige und ältere Frauen aus der Oberschicht. Dennoch wird deutlich, dass bei Männern das Aufhörverhalten vor allem im mittleren Lebensalter mit der Schichtzugehörigkeit variiert. Ab dem Alter 50 sind diesbezüglich keine Unterschiede mehr erkennbar. Bei Frauen zeigen sich hingegen bis ins hohe Alter schichtspe- zifische Ausprägungen des Ausstiegverhaltens, was dafür spricht, dass bei Männern und Frauen andere Mechanismen für den Einfluss der Schichtzugehörigkeit auf die Aufhörbereitschaft verantwortlich sind.

\section{Bildungs-, Einkommens- und Berufsstatusdifferenzen in den Rauchgewohnheiten}

Weiteren Aufschluss über die soziale Ungleichheit des Rauchverhaltens geben Analysen, die sich an den Einzelindikatoren Schulbildung, Äquivalenzeinkommen und beruflicher Stellung ausrichten. Diesen zufolge rauchen Männer mit niedriger oder mittlerer Schulbildung häufiger als Männer mit hoher Schulbildung (• Tabelle 3). Besonders deutlich zeigt sich dies im jungen und mittleren Alter. Darüber hinaus 
Tabelle 4

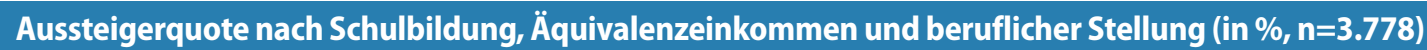

\begin{tabular}{|c|c|c|c|c|c|c|c|c|c|c|c|c|}
\hline & \multicolumn{6}{|c|}{ Männer } & \multicolumn{6}{|l|}{ Frauen } \\
\hline & $18-29$ & $30-39$ & $40-49$ & $50-59$ & $60-79$ & Gesamt & $18-29$ & $30-39$ & $40-49$ & $50-59$ & $60-79$ & Gesamt \\
\hline \multicolumn{13}{|l|}{ Schulbildung } \\
\hline Volks- oder Haupt- & 11,5 & 22,1 & 39,6 & 52,4 & 72,3 & 47,0 & 10,4 & 24,8 & 38,5 & 41,6 & 42,7 & 33,9 \\
\hline schule & 11,1 & 30,2 & 45,9 & 56,2 & 78,9 & 39,0 & 10,8 & 34,0 & 42,9 & 51,7 & 57,6 & 33,4 \\
\hline $\begin{array}{l}\text { Mittlere Reife } \\
\text { Abitur }\end{array}$ & 12,5 & 25,6 & 44,3 & 51,3 & 79,8 & 40,6 & 19,1 & 45,2 & 53,7 & 61,8 & $(69,2)$ & 43,1 \\
\hline$\chi^{2}$-Test, Signifikanz & n.s. & n.s. & n.s. & n.s. & n.s. & $p<0,05$ & n.s. & $p<0,01$ & n.s. & n.s. & $p<0,05$ & $p<0,05$ \\
\hline \multicolumn{13}{|c|}{ Äquivalenzeinkommen } \\
\hline$<60 \%$ & 9,2 & 27,9 & 35,6 & 40,7 & 59,6 & 32,4 & 7,1 & 26,7 & 34,5 & $(20,0)$ & $(63,6)$ & 23,5 \\
\hline $60-150 \%$ & 16,1 & 27,4 & 43,6 & 54,8 & 77,0 & 45,8 & 18,4 & 32,2 & 45,9 & 50,9 & 49,2 & 38,6 \\
\hline$>150 \%$ & $(3,7)$ & 23,2 & 57,1 & 58,4 & 79,7 & 50,0 & $(11,1)$ & 50,0 & 40,0 & 47,6 & $(76,9)$ & 44,4 \\
\hline$\chi^{2}$-Test, Signifikanz & n.s. & n.s. & $p<0,05$ & n.s. & $p<0,05$ & $p<0,001$ & n.s. & $p<0,05$ & n.s. & $\mathrm{p}<0,05$ & n.s. & $p<0,001$ \\
\hline \multicolumn{13}{|l|}{ Berufliche Stellung } \\
\hline Niedrig & 14,3 & 20,5 & 35,4 & 46,6 & 66,7 & 37,7 & 11,8 & 19,8 & 24,1 & 35,7 & 40,0 & 25,5 \\
\hline Einfach & 13,7 & 29,9 & 32,2 & 44,7 & 74,8 & 39,5 & 8,3 & 29,1 & 43,2 & 45,7 & 41,2 & 33,0 \\
\hline Mittel & 18,6 & 26,9 & 58,7 & 64,3 & 78,3 & 52,3 & 17,2 & 37,2 & 49,6 & 50,6 & 69,1 & 42,3 \\
\hline Höher & $(7,1)$ & 28,4 & 39,9 & 53,9 & 75,7 & 49,2 & $(7,1)$ & 44,6 & 41,7 & 53,3 & $(51,9)$ & 44,1 \\
\hline$\chi^{2}$-Test, Signifikanz & n.s. & n.s. & $p<0,01$ & $p<0,05$ & n.s. & $p<0,001$ & n.s. & $p<0,01$ & $p<0,05$ & n.s. & $p<0,01$ & $p<0,001$ \\
\hline
\end{tabular}

() Fallzahl $<30$.

weisen die Ergebnisse für Männer ein niedriges Einkommen und einen niedrigen Berufsstatus als Bestimmungsgrößen des Rauchens aus. Im mittleren Lebensalter raucht ungefähr die Hälfte der Männer der niedrigsten Einkommensgruppe, aber lediglich ein Viertel bis ein Drittel der Männer mit den höchsten Einkommen. Ähnliche Unterschiede zeigen sich in Bezug auf die berufliche Stellung: Im mittleren Lebensalter finden sich die höchsten Raucherprävalenzen in der niedrigsten Berufsstatusgruppe, also bei un- und angelernten Arbeitern. Allerdings zeigt sich kein lineares Gefälle, da der Anteil der Raucher in der höheren Statusgruppe zum Teil noch über dem entsprechenden Anteil in der mittleren Statusgruppe liegt.

Bei Frauen lassen sich nur bis zum Alter von 40 Jahren bedeutsame Bildungs-, Einkommens- und Berufsstatusunterschiede im Rauchverhalten beobachten. Ausschlaggebend hierfür dürfte der bereits angesprochene Kohorteneffekt sein, der sich an einem hohen Anteil älterer Frauen, die nie geraucht haben, festmachen lässt. Daneben könnte die geringere Bildungs- und Erwerbsbeteiligung von Frauen der älteren Geburtskohorten zum Tragen kommen, da dadurch die soziale Ungleichheit, insbesondere wenn sie über In- dividualmerkmale erhoben wird, schwächer ausfällt.

Im Ausstiegsverhalten treten bei Männern nur schwache Unterschiede nach Schulbildung und Berufsstatus zutage (- Tabelle 4). Ein niedriges Einkommen wirkt sich hingegen im mittleren und höheren Alter nachteilig auf die Beendigung des Rauchens aus. Die Ergebnisse für Frauen deuten darauf hin, dass eine niedrige Schulbildung und ein niedriger Berufsstatus die Aussicht auf eine Tabakentwöhnung verringern. Auch Frauen mit niedrigem Einkommen geben seltener das Rauchen wieder auf.

\section{Für das Ausstiegsverhalten von Männern scheint das Einkommen, für das von Frauen vor allem die Schulbildung von Bedeutung zu sein}

Eine abschließende Bewertung des Einflusses von Bildung, Einkommen und Berufsstatus auf das Rauchverhalten erschließt sich erst durch eine multivariate Betrachtung. Dazu wurden logistische Regressionen für das aktuelle Rauchen und die Beendigung des Tabakkonsums durchgeführt. In beiden Fällen wurde zunächst der Einfluss der Prädiktoren separat bestimmt
(Modell 1), bevor sie in ein gemeinsames Modell aufgenommen wurden (Modell 2), um ihre relative Bedeutung zu ermitteln. Dabei wurde neben dem Alterseffekt für weitere relevante Drittvariablen wie Familienstand, Haushaltsform, Erwerbsstatus, Wohnregion und chronische Krankheit kontrolliert.

- Tabelle 5 gibt zunächst die Ergebnisse für das aktuelle Rauchverhalten wieder. Ausgewiesen sind Odds Ratios (OR) mit den dazugehörigen Konfidenzintervallen (KI 95\%). Die Odds Ratios besagen, wie hoch die Chance ist, dass die Angehörigen der betrachteten Bildungs-, Einkommens- und Berufsstatusgruppen rauchen, im Verhältnis zur Chance in der jeweiligen Referenzkategorie (Ref.). Entsprechend Modell 1 üben bei Frauen nur die Schulbildung und eine niedrige berufliche Stellung einen bedeutsamen Einfluss auf das Rauchverhalten aus; bei Männern ist außerdem ein Einkommenseffekt zu verzeichnen. Durch die gemeinsame Betrachtung der Prädiktoren in Modell 2 lassen sich die Ergebnisse präzisieren: Im Vergleich zu Frauen mit Abitur ist die Chance zu rauchen bei Frauen mit niedriger Schulbildung um den Faktor 2,3 und bei Frauen mit mittleren Schulbildung um den Faktor 1,7 erhöht. Bei Männern fällt 
Tabelle 5

Einfluss von Schulbildung, Äquivalenzeinkommen und beruflicher Stellung auf das aktuelle

Rauchverhalten. Ergebnisse logistischer Regressionen bei statistischer Kontrolle von Alter, Familienstand, Erwerbsstatus, Wohnregion und chronischer Krankheit (Odds Ratios mit Konfidenzintervallen, n=6.963)

\begin{tabular}{|c|c|c|c|c|c|c|c|c|}
\hline & \multicolumn{4}{|c|}{ Männer } & \multicolumn{4}{|c|}{ Frauen } \\
\hline & \multicolumn{2}{|c|}{ Modell 1} & \multicolumn{2}{|c|}{ Modell 2} & \multicolumn{2}{|c|}{ Modell 1} & \multicolumn{2}{|c|}{ Modell 2} \\
\hline & OR & KI 95\% & OR & KI 95\% & OR & KI 95\% & OR & KI 95\% \\
\hline \multicolumn{9}{|l|}{ Schulbildung } \\
\hline Volks- oder Hauptschule & 1,76 & $1,45-2,13$ & 1,62 & $1,30-2,02$ & 2,26 & $1,79-2,86$ & 2,32 & $1,79-3,01$ \\
\hline Mittlere Reife & 1,40 & $1,14-1,71$ & 1,36 & $1,10-1,68$ & 1,59 & $1,27-1,99$ & 1,71 & $1,35-2,17$ \\
\hline Abitur & Ref. & - & Ref. & - & Ref. & - & Ref. & - \\
\hline \multicolumn{9}{|l|}{ Äquivalenzeinkommen } \\
\hline$<60 \%$ & 1,57 & $1,19-2,08$ & 1,31 & $0,97-1,77$ & 1,06 & $0,76-1,47$ & 0,80 & $0,57-1,01$ \\
\hline $60-150 \%$ & 1,10 & $0,86-1,39$ & 1,02 & $0,79-1,31$ & 0,88 & $0,66-1,18$ & 0,74 & $0,55-1,00$ \\
\hline$>150 \%$ & Ref. & - & Ref. & - & Ref. & - & Ref. & - \\
\hline \multicolumn{9}{|l|}{ Berufliche Stellung } \\
\hline Niedrig & 1,66 & $1,30-2,12$ & 1,24 & $0,94-1,63$ & 1,43 & $1,07-1,93$ & 1,01 & $0,72-1,40$ \\
\hline Einfach & 1,16 & $0,95-1,42$ & 0,88 & $0,70-1,11$ & 1,07 & $0,80-1,41$ & 0,77 & $0,57-1,05$ \\
\hline Mittel & 0,83 & $0,68-1,03$ & 0,72 & $0,58-0,91$ & 0,81 & $0,62-1,05$ & 0,67 & $0,51-0,88$ \\
\hline Höher & Ref. & - & Ref. & - & Ref. & - & Ref. & - \\
\hline
\end{tabular}

OR Odds Ratio; KI Konfidenzintervall; Ref. Referenzkategorie.

a In Modell 1 werden die Prädiktoren Schulbildung, Äquivalenzeinkommen und berufliche Stellung separat und in Modell 2 gemeinsam betrachtet.

Tabelle 6

Einfluss von Schulbildung, Äquivalenzeinkommen und beruflicher Stellung auf das Ausstiegsverhalten.

Ergebnisse logistischer Regressionen bei statistischer Kontrolle von Alter, Familienstand, Erwerbsstatus, Wohnregion und chronischer Krankheit (Odds Ratios mit Konfidenzintervallen, $\mathrm{n}=3.778)^{\mathrm{a}}$

\begin{tabular}{|c|c|c|c|c|c|c|c|c|}
\hline & \multicolumn{4}{|c|}{ Männer } & \multicolumn{4}{|c|}{ Frauen } \\
\hline & \multicolumn{2}{|c|}{ Modell 1} & \multicolumn{2}{|c|}{ Modell 2} & \multicolumn{2}{|c|}{ Modell 1} & \multicolumn{2}{|c|}{ Modell 2} \\
\hline & OR & KI 95\% & OR & KI 95\% & OR & KI 95\% & OR & KI 95\% \\
\hline \multicolumn{9}{|l|}{ Schulbildung } \\
\hline Volks- oder Hauptschule & Ref. & - & Ref. & - & Ref. & - & Ref. & - \\
\hline Mittlere Reife & 1,29 & $1,02-1,65$ & 1,20 & $0,93-1,54$ & 1,76 & $1,33-2,34$ & 1,50 & $1,10-2,02$ \\
\hline Abitur & 1,16 & $0,90-1,49$ & 1,12 & $0,84-1,51$ & 2,83 & $2,06-3,89$ & 2,38 & $1,67-3,34$ \\
\hline \multicolumn{9}{|l|}{ Äquivalenzeinkommen } \\
\hline$<60 \%$ & Ref. & - & Ref. & - & Ref. & - & Ref. & - \\
\hline $60-150 \%$ & 1,44 & $1,11-1,88$ & 1,34 & $1,02-1,76$ & 1,45 & $1,05-2,01$ & 1,27 & $0,91-1,77$ \\
\hline$>150 \%$ & 1,52 & $1,06-2,02$ & 1,49 & $1,00-2,21$ & 1,69 & $1,07-2,67$ & 1,11 & $0,68-1,80$ \\
\hline \multicolumn{9}{|l|}{ Berufliche Stellung } \\
\hline Niedrig & Ref. & - & Ref. & - & Ref. & - & Ref. & - \\
\hline Einfach & 1,16 & $0,85-1,58$ & 1,12 & $0,82-1,54$ & 1,54 & $1,06-2,25$ & 1,38 & $0,94-2,04$ \\
\hline Mittel & 1,82 & $1,32-2,51$ & 1,62 & $1,16-2,26$ & 2,47 & $1,74-3,50$ & 1,81 & $1,24-2,64$ \\
\hline Höher & 1,22 & $0,90-1,66$ & 1,03 & $0,73-1,45$ & 2,38 & $1,56-3,61$ & 1,51 & $0,95-2,34$ \\
\hline
\end{tabular}

OR Odds Ratio; KI Konfidenzintervall; Ref. Referenzkategorie.

${ }^{a}$ In Modell 1 werden die Prädiktoren Schulbildung, Äquivalenzeinkommen und berufliche Stellung separat und in Modell 2 gemeinsam betrachtet.

der Bildungseffekt etwas geringer aus, ist aber bei einem Odds Ratio von 1,6 in der niedrigsten und 1,4 in der mittleren im Vergleich zur höchsten Bildungsgruppe signifikant. Der Einfluss des Einkommens und des Berufsstatus erweist sich bei gleichzei- tiger Berücksichtigung der Bildung weder bei Männern noch bei Frauen als statistisch bedeutsam.

Die Ergebnisse zum Ausstiegsverhalten sprechen dafür, dass bei Männern die Chance, mit dem Rauchen aufzuhören, im mittleren und höheren Einkommensbereich höher ist als im unteren Einkommensbereich (• Tabelle 6). Von der Schulbildung und dem Berufsstatus geht hingegen nur ein geringer Einfluss aus mit der Ausnahme, dass Männer in mittlerer beruf- 
licher Stellung im Vergleich zu Männern mit niedrigem Berufsstatus häufiger das Rauchen aufgeben. Für das Ausstiegsverhalten von Frauen scheint vor allem die Schulbildung von Bedeutung zu sein. Im Vergleich zu Frauen mit Volks- oder Hauptschulabschluss hören Frauen mit mittlerer Reife oder Abitur 1,5- bzw. 2,4-mal häufiger mit dem Rauchen auf. Außerdem schaffen wie bei Männern vor allem Frauen mit mittlerem Berufsstatus von der $\mathrm{Zi}$ garette loszukommen.

\section{Diskussion}

Die Ergebnisse des Bundes-Gesundheitssurveys 1998 bestätigen die aus früheren Studien bekannten sozialen Unterschiede im Rauchverhalten der erwachsenen Bevölkerung in Deutschland, verdeutlichen aber zugleich, dass sich nur im mittleren Lebensalter ein stark ausgeprägtes soziales Gefälle abzeichnet. Im jüngeren Erwachsenenalter ist der Anteil der Raucher in allen sozialen Gruppen verhältnismäßig hoch, während sich im fortgeschrittenen Lebensalter generell ein starker Rückgang zeigt, der auf die Bedeutung alterskorrelierter Veränderungen der Lebensweise und der Gesundheit hinweist, aber auch durch vorzeitige Todesfälle langjähriger Raucher mitbestimmt wird. Darüber hinaus sprechen die erzielten Befunde gegen die verbreitete Vorstellung, dass das Rauchen bei Frauen im Vergleich zu Männern im geringeren Maß mit dem Sozialstatus variiert. Zumindest für die jüngeren Geburtskohorten lässt sich feststellen, dass der Zusammenhang zwischen der Position im gesellschaftlichen Ungleichheitsgefüge und dem Tabakkonsum bei Männern und Frauen ähnlich stark ausgeprägt ist. Von den einbezogenen Sozialindikatoren übt die Schulbildung den stärksten Einfluss auf die Rauchgewohnheiten aus, was bei Frauen auch in Bezug auf das Ausstiegsverhalten beobachtet werden kann. Die Bedeutung der Einkommenssituation ist vergleichsweise gering. Allerdings geben Männer der mittleren und höheren Einkommensgruppen häufiger das Rauchen auf als Männer mit niedrigem Einkommen. Wahrscheinlich würden die Einkommenseffekte stärker ausfallen, wenn die Abgrenzung des Niedrigeinkommensbzw. Armutsbereichs bei $40 \%$ oder 50\% statt bei 60\% des gesellschaftlichen Durchschnittseinkommens ansetzen würde, was im Bundes-Gesundheitssurvey 1998 aufgrund zu niedriger Fallzahlen jedoch nicht möglich ist. Ebenso ist anzunehmen, dass der Einfluss der Arbeitswelt und des Berufs auf das Rauchen deutlicher zum Ausdruck käme, wenn neben der beruf lichen Stellung auch die berufliche Tätigkeit und Berufsgruppenzugehörigkeit betrachtet würde. Auch hier sind Auswertungen auf Basis des Bundes-Gesundheitssurveys 1998 durch die Fallzahlen Grenzen gesetzt. Ein angemessener Zugang eröff net sich über die alle 4 Jahre durchgeführte Mikrozensus-Zusatzerhebung zur Gesundheit, die auch regelmäßig für berufsgruppenspezifische Analysen des Rauchverhaltens herangezogen wird $[13,15]$.

\section{(D) Der Befund sozialer Unterschie- de im Rauchverhalten deutet auf noch unzureichend ausge- schöpfte Präventions- und Interventionspotenziale hin}

Trotz dieser Einschränkungen lassen die Ergebnisse keinen Zweifel daran, dass durch eine nachhaltige Verringerung des Tabakkonsums eine Verbesserung und gerechtere Verteilung der Gesundheitschancen in der Bevölkerung erreicht werden könnte. Der Befund sozialer Unterschiede im Rauchverhalten beschreibt somit nicht nur ein gesundheitspolitisches Problem, sondern deutet zugleich auf noch unzureichend ausgeschöpfte Präventionsund Interventionspotenziale hin. Aus diesem Grund gehört das Rauchen zu den Themenfeldern, zu denen im Projekt "gesundheitsziele.de" gesundheitspolitische Handlungsvorgaben definiert und Maßnahmen zu deren Umsetzung entwickelt werden [4]. Die bislang formulierten Zielvorstellungen und Empfehlungen beziehen sich zuvorderst auf die Förderung des Nichtrauchens, die Raucherentwöhnung und den Schutz vor Passivrauchen. Die Verringerung der gesundheitlichen Chancenungleichheit wird dabei als wichtige Querschnittsaufgabe angesehen, die sowohl bei der Entwicklung als auch bei der Umsetzung und Evaluation der Zielvorstellungen $\mathrm{zu}$ berücksichtigen ist.

$\mathrm{Da}$ in einigen Ländern bereits umfassende Programme zur Eindämmung des Tabakkonsums durchgeführt werden, kann auf Erkenntnisse über die Wirksamkeit von Interventionen zurückgegriffen werden. Zu den gut bewährten Maßnahmen zählen: Erhöhungen der Tabaksteuer, Bekämpfung des Zigarettenschmuggels, Werbeverbote für Tabakprodukte, Rauchverbote in öffentlichen Einrichtungen und am Arbeitsplatz, Abgabeverbote an Kinder und Jugendliche, schulische, freizeit- und gemeindebezogene Maßnahmen der Tabakprävention, flächendeckende massenmediale Aufklärungs- und Informationskampagnen sowie personalkommunikative Beratungs- und Behandlungsangebote für unterschiedliche Zielgruppen. Eine deutliche Reduzierung des Tabakkonsums und des tabakassoziierten Krankheits- und Sterbegeschehens lässt sich offenbar vor allem mit einem Maßnahmenbündel erreichen, das bevölkerungsbezogene und individuumszentrierte Vorgehensweisen kombiniert und aufeinander abstimmt [27].

Eine erfolgreiche Umsetzung wirksamkeitsgeprüfter Interventionen bedeutet aber nicht zwangsläufig, dass sich die sozialen Unterschiede im Rauchverhalten verringern. Viele Maßnahmen erreichen eher die sozial Bessergestellten und damit die Gruppen, in denen bereits weniger geraucht wird. Dies dürfte besonders für massenmediale Aufklärungskampagnen, settingbezogene Präventionsstrategien und auch Beratungs- und Behandlungsangebote zur Tabakentwöhnung gelten. Von einem umfassenden Tabakwerbeverbot würden wahrscheinlich auch die sozial benachteiligten Bevölkerungsgruppen profitieren, zumal sie eine der hauptsächlichen Zielgruppen der Tabakwerbung darstellen. Auch von Tabaksteuererhöhungen wird sich neben einer rückläufigen Gesamtnachfrage eine Verringerung der sozialen Unterschiede im Rauchverhalten versprochen. Die internationalen Erfahrungen legen nahe, dass die einkommensschwächeren Bevölkerungsgruppen sensibler auf Preiserhöhungen reagieren [28]. Wenn weiter geraucht wird, ergibt sich jedoch eine höhere finanzielle Belastung und häufig eine Einschränkung bei notwendigen $\mathrm{Be}$ darfsgütern wie Nahrung, Kleidung oder Hygieneprodukten. Für Tabaksteuererhöhungen gilt somit, wie für die meisten anderen Maßnahmen der Tabakprävention 
und Tabakkontrollpolitik, dass die Möglichkeit unterschiedlicher Wirkungen auf verschiedene Bevölkerungsgruppen mitbedacht werden muss, damit sie sich nicht entgegen der eigentlichen Intention in einer Ausweitung der sozialen Unterschiede im Rauchverhalten und der daraus resultierenden gesundheitlichen Chancenungleichheit niederschlagen [29].

\section{Korrespondierender Autor}

\section{T. Lampert}

Abt. Epidemiologie

und Gesundheitsberichterstattung,

Robert Koch-Institut,

Nordufer 20, 13353 Berlin

E-Mail: t.lampert@rki.de

\section{Literatur}

1. Mielck A (2000) Soziale Ungleichheit und Gesundheit. Empirische Ergebnisse, Erklärungsansätze, Interventionsmöglichkeiten. Hans Huber, Bern Göttingen Toronto Seattle

2. Helmert U (2003) Soziale Ungleichheit und Krankheitsrisiken. Maro, Augsburg

3. World Health Organization (1999) Health 21. The health for all policy framework for the WHO European Region. WHO, Copenhagen

4. Bundesministerium für Gesundheit und Soziale Sicherung (2003) gesundheitsziele.de. Forum zur Entwicklung und Umsetzung von Gesundheitszielen in Deutschland. BMGS, Bonn

5. Steinkamp G (1993) Soziale Ungleichheit, Erkrankungsrisiko und Lebenserwartung: Kritik der sozialepidemiologischen Ungleichheitsforschung. Sozial Präventivmed 38:111-122

6. Elkeles T, Mielck A (1997) Entwicklung eines Modells zur Erklärung gesundheitlicher Ungleichheit. Gesundheitswesen 59:137-143

7. International Agency for Research on Cancer (2004) IARC monographs on the evaluation of the carcinogenic risks to humans. Tobacco smoke and involuntary smoking. International Agency for Research on Cancer, Lyon

8. US Department of Health and Human Services (2004) The health consequences of smoking: a report of the surgeon general. US Department of Health and Human Services, Centers for Disease Control and Prevention, National Center for Chronic Disease Prevention and Health Promotion, Office on Smoking and Health, Washington, D.C.

9. Ezzati M, Lopez AD (2003) Estimates of global mortality attributable to smoking in 2000 . Lancet 362:847-852

10. John U, Hanke M (2001) Tabakrauch - attributable Mortalität in den deutschen Bundesländern. Gesundheitswesen 63:363-369

11. Härtel U, Stieber J, Keil U (1993) Der Einfluss von Ausbildung und beruflicher Position auf Veränderungen im Zigarettenrauchen und Alkoholkonsum: Ergebnisse der MONICA Augsburg Kohortenstudie. Sozial Präventivmed 38:133-141

12. Heinemann L, Helmert U, Classen E, Greiser E (1996) Social gradient of CVD Risk in Germany before/after unification. Rev Environment Health 11:7-14
13. Helmert U, Borgers D (1998) Rauchen und Beruf Eine Analyse von 100.000 Befragten des Mikrozensus 1995. Bundesgesundheitsblatt 41:102-107

14. Helmert U, Maschewsky-Schneider U (1998) Zur Prävalenz des Tabakrauchens bei Arbeitslosen und Armen. In: Henkel D, Vogt I (Hrsg) Sucht und Armut. Alkohol, Tabak, Medikamente, illegale Drogen. Leske \& Budrich, Opladen, S 153-165

15. Helmert U, Borgers D, Bammann K (2001) Soziale Determinanten des Rauchverhaltens in Deutschland: Ergebnisse des Mikrozensus 1995. Sozial Präventivmed 46:172-181

16. Helmert U, Lang P, Cuelenaere B (1998) Rauchverhalten von Schwangeren und Müttern mit Kleinkindern. Sozial- und Präventivmedizin 43:51-58

17. Thefeld W (2002) Rauchen und Kinder: Rauchverhalten von Frauen im gebährfähigen Alter, von Eltern und Heranwachsenden. In: Robert Koch-Institut (Hrsg) Der Bundes-Gesundheitssurvey - Baustein der Gesundheitssurveillance in Deutschland. RKI, Berlin, S 27-29

18. Helmert U, Buitkamp M (2004) Die Veränderung des Rauchverhaltens in Deutschland von 1985 bis 2002. Gesundheitswesen 66:102-106

19. Stolzenberg $H$ (2002) Gesundheitssurveys in Deutschland. In: Robert Koch-Institut (Hrsg) Der Bundes-Gesundheitssurvey - Baustein der Gesundheitssurveillance in Deutschland. RKI, Berlin, S 22-24

20. Thelefeld W, Stolzenberg H, Bellach BM (1999) Bundes-Gesundheitssurvey: Response, Zusammensetzung der Teilnehmer und Non-Responder-Analyse. Gesundheitswesen 61 [Sonderheft 2]:57-61

21. Bellach BM, Knopf H, Thefeld W (1998) Der Bundes Gesundheitssurvey 1997/98. Gesundheitswesen 60 [Sonderheft 2]:59-68

22. Winkler J, Stolzenberg H (1999) Der Sozialschichtindex im Bundes-Gesundheitssurvey. Gesundheitswesen 61 [Sonderheft 2]:178-183

23. Hanesch W, Krause P et al. (2000) Armut und Ungleichheit in Deutschland. Der neue Armutsbericht der Hans-Böckler-Stiftung, des DGB und des Paritätischen Wohlfahrtverbandes. Rowohlt Taschenbuch, Reinbek bei Hamburg

24. Hoffmeyer-Zlotnik (1998) "Beruf" und "berufliche Stellung" als Indikatoren für soziale Schichtung. In: Ahrens W, Bellach BM, Jöckel KH (Hrsg) Messung soziodemographischer Merkmale in der Epidemiologie. RKI-Schriften 1/98, MMV Medizin Verlag GmbH, München, S 54-64

25. Lundberg O, Kareholt I (1996) The social patterning of mortality in a cohort of elderly Swedes. Yearbook Population Res Finland 33:101-110

26. Marmot MG, Shipley MJ (1996) Do socioeconomic differences in mortality persist after retirement? 25 year follow up of civil servants from the first Whitehall study. Br Med J 313:1177-1180

27. Pott E, Lang P, Töppich J (2003) Gesundheitsziel: Tabakkonsum reduzieren. Bundesgesundheitsblatt Gesundheitsforschung Gesundheitsschutz 46:150 155

28. Chaloupka FJ, Hu H, Warner KE et al. (2000) The taxation of tobacco products. In: Jha P, Chaloupka FJ (eds) Tobacco control in developing countries. Oxford University Press, New York, pp 237-272

29. Schulze A, Deutsches Krebszentrum (2004) Rauchen und soziale Ungleichheit - Konsequenzen für die Tabakkontrollpolitik. Heidelberg

\section{Hygiene-Preis der Rudolf Schüle Stiftung}

\section{Der Preis wird im Jahr 2005 für hervorragende wissenschaftliche Untersuchungen auf den Gebieten HYGIENE - MIKROBIOLOGIE - PRÄVENTIVMEDIZIN ausgeschrieben.}

Erwünscht sind Arbeiten, die sich mit der Prävention und Kontrolle übertragbarer Erkrankungen befassen.

Berücksichtigt werden insbesondere Arbeiten zur Entwicklung und Anwendung von Präventionsstrategien und von antimikrobiell bzw. antiviral wirksamen Stoffen und Verfahren zur Antiseptik, Desinfektion und Konservierung.

Arbeiten über den Zusammenhang zwischen solchen Anwendungen und Fragen der Epidemiologie sowie der Ökologie gesundheitlich relevanter Mikroorganismen und über Immunprophylaxe sind ebenso erwünscht wie Arbeiten über Konsequenzen für das Gesundheitswesen und die Gesundheitsökonomie.

Eingereicht werden können Arbeiten, die nach dem 1. Januar 2003 in einer wissenschaftlichen Zeitschrift oder einer Schriftenreihe in deutscher oder englischer Sprache erschienen sind bzw. über deren Annahme eine Bescheinigung des Herausgebers vorgelegt wird.

Der Preis ist dotiert mit $€ 15.000$,-- und wird im Rahmen einer Feierstunde an eine Einzelpersönlichkeit oder an eine Gruppe verliehen. In Ausnahmefällen kann der Preis geteilt werden. Über die Verleihung entscheidet ein Preisrichterkollegium, dessen Beschluss unanfechtbar ist. Der Rechtsweg bleibt ausgeschlossen.

Bewerber möchten ihre Arbeit in 10-facher Ausfertigung bis zum 15. Februar 2005 (Poststempel) an das Sekretariat der Rudolf Schülke Stiftung, Robert-KochStr. 2, 22851 Norderstedt, einreichen. Die Fachvertreter an den Universitäten und entsprechenden Institutionen können Vorschläge für die Verleihung des Preises an die gleiche Adresse richten. 